\title{
Prediction Model of Toxicity of Anilines and Phenols in Leather
}

\section{Chemicals}

\author{
Xihua Du ${ }^{\mathrm{a}}$, Jun Zhou ${ }^{\mathrm{b}}$ and Lin $\operatorname{Tian}^{\mathrm{c}}$ \\ School of Chemistry and Chemical Engineering , Xuzhou Institute of Technology , Xuzhou, Jiangsu, \\ 221111, China

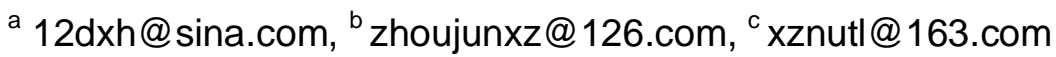

Keywords : Quantitative Structure-Activity Relationships(QSAR), Aniline, Phenol, Biological Toxicity, Structure Index

\begin{abstract}
In order to analyze the relationship between structural characteristics and toxicity of aniline and phenolic compounds in leather chemicals, the molecular connectivity indices, the molecular shape indices, electrical topology status relationship indices and electrical distance vectors of 36 kinds of aniline and phenolic compounds were calculated. Quantitative structure-activity relationship (QSAR) model was established by building multiple linear regression equation. The $\mathrm{R}_{\text {adj }}^{2}$ of the equation was 0.885 and it showed good predictive ability. Therefore, our model had good predictive ability of biological toxicity of aniline and phenols in leather chemicals.
\end{abstract}

\section{Introduction}

Anilines and Phenols are the most common compounds in Chemical and Medical industries. They are widely used in the producing and handing process of leather. They are leather chemicals themselves [1] and they are also used for composing other leather chemicals. In the producing process of leather, p-phenylenediamine is not only the chemical intermediate of synthetic dye but also a common oxidation base [2]. However, aniline and phenolic compounds are pollutants to the environment, and they are also toxic to organism. For instance, p-phenylenediamine has very strong sensitization and it may cause diseases such as contact dermatitis and bronchial asthma[3]. Phenolic compounds make protein denaturated or precipitated, thus, it can not only erode skin and mucosa but also cause damage to liver and kidney. Phenolic compound is teratogenic, carcinogenic and mutagenic, so that many countries list it as one of the key pollutants[4,5]. Due to the vast variety of aniline and phenolic compounds, huge amounts of time and money would be poured into if their toxicity is only examined by the experimental method. Moreover, there are no standard samples for some compounds so that it is difficult to do the cross-check analysis. Therefore, it is practical to use the QSAR method which has been widely used in chemical, environmental, medical and food industries [6,7] to predict the toxicity of anilines and phenols.

After a lot of research work, researchers found that biological activity of unknown compounds can be predicted by using statistical regression analysis method to build the model about structural characteristics of compounds and their activities. Namely, by building QSAR model, the activity, toxicity, degradability and physicochemical properties of environmental pollutants can be predicted. The model helps significantly reduce biological toxicity experiments which reduces experimental cost and also covers the shortage of missing data of standard samples for some compounds. For 
these reasons, QSAR method has been gradually applied in the prediction of compound toxicity and activity along with environmental risk assessment. [8,9].

In this paper, molecular structure of anilines and phenols were constructed by program. Structural indices were calculated and six indices were optimized selected in MATLAB. The six indices, along with the toxicity of aniline and phenolic compounds in leather chemicals to daphnia magna $\left(p \mathrm{EC}_{50}\right)$ were analyzed by regression analysis and then the QSAR model was established. The predicted outcomes of the model were good and it would contribute a lot if the model could be applied in environmental risk assessment.

\section{Calculation of structural indices of anilines and phenols}

Firstly, the molecular structure and the toxicity of 36 anilines and phenolic compounds in leather material listed in the reference [10] to daphnia magna $p \mathrm{EC}_{50}$ (negative logarithm of $24 \mathrm{~h}$ inhibitory concentration 50\% ) were analyzed. Molecular structures of 36 anilines and phenolic compounds were constructed by the software Chem 3D. Then, molecular connectivity indices, molecular shape indices, topological state indices and molecular electronegativity distance vectors of 36 anilines and phenolic compounds were calculated by the self-designed program [11,12] in MATLAB. Three molecular shape indices $K_{1}, K_{2}$ and $K_{4}$, along with three topological state indices $E_{13}, E_{16}$ and $E_{17}$ were optimized selected. The QSAR model was established by linear regression analysis of the six selected indices and $p \mathrm{EC}_{50}$.

\section{Prediction of the toxicity of aniline and phenolic compounds to daphnia magna}

The six optimized selected indices and the toxicity of compounds to daphnia magna were analyzed by the linear regression using SPSS software. The QSAR model was established as:

$$
\begin{gathered}
p \mathrm{EC}_{50}=4.363+0.280 K_{1}-1.386 K_{2}+2.174 K_{4}-0.0134 E_{13}-0.0180 E_{16}-0.0795 E_{17} \quad(1) \\
\mathrm{N}=36, \quad \mathrm{R}=0.951 \quad \mathrm{R}_{\text {adj }}^{2}=0.885 \quad \mathrm{~S}=0.239 \quad \mathrm{~F}=45.977
\end{gathered}
$$

In this equation, $\mathrm{N}$ was the sample size, $\mathrm{R}$ was the correlation coefficient, $\mathrm{R}^{2}$ adj was coefficient of determination, $\mathrm{S}$ was standard error and $\mathrm{F}$ was Fischer ratio. The correlation coefficient was 0.951 which meant there was good correlation among the indices. The estimated value $p \mathrm{EC}_{50}$ was close to experimental value. Their mean absolute error was only 0.16 and the average relative error was $3.94 \%$. The relevant data can be seen in Table 1 . 
Table. 1 Comparison between Experimental Values and Estimated Values of $p \mathrm{EC}_{50}$

\begin{tabular}{|c|c|c|c|c|c|c|c|c|c|}
\hline \multirow{2}{*}{ No } & \multirow{2}{*}{$K_{1}$} & \multirow{2}{*}{$K_{2}$} & \multirow{2}{*}{$K_{4}$} & \multirow{2}{*}{$E_{13}$} & \multirow{2}{*}{$E_{16}$} & \multirow{2}{*}{$E_{17}$} & \multicolumn{3}{|c|}{$p E C_{50}$} \\
\hline & & & & & & & Exp. & Cal. & error \\
\hline 1 & 2.646 & 1.968 & 0.742 & 0 & 0 & 5.427 & 3.42 & 3.56 & 0.14 \\
\hline 2 & 2.853 & 2.159 & 0.869 & 0 & 0 & 5.368 & 3.64 & 3.63 & -0.01 \\
\hline 3 & 3.814 & 2.564 & 1.271 & 0 & 0 & 5.398 & 4.26 & 4.21 & -0.05 \\
\hline 4 & 3.814 & 2.564 & 1.271 & 0 & 0 & 5.404 & 4.47 & 4.21 & -0.26 \\
\hline 5 & 4.833 & 2.971 & 1.678 & 0 & 0 & 5.428 & 4.86 & 4.81 & -0.05 \\
\hline 6 & 2.990 & 2.286 & 0.956 & 0 & 0 & 5.406 & 3.81 & 3.68 & -0.13 \\
\hline 7 & 6.168 & 4.461 & 3.132 & 25.780 & 31.741 & 5.249 & 5.45 & 5.38 & -0.07 \\
\hline 8 & 3.539 & 2.332 & 1.102 & 0 & 0 & 5.276 & 4.23 & 4.10 & -0.13 \\
\hline 9 & 3.338 & 2.656 & 1.339 & 8.402 & 10.258 & 5.320 & 3.91 & 3.81 & -0.10 \\
\hline 10 & 3.338 & 2.656 & 1.339 & 8.370 & 10.214 & 5.320 & 3.87 & 3.81 & -0.06 \\
\hline 11 & 4.745 & 3.558 & 2.232 & 16.962 & 20.792 & 5.287 & 5.24 & 4.59 & -0.65 \\
\hline 12 & 4.274 & 3.061 & 1.749 & 8.417 & 10.282 & 5.355 & 4.13 & 4.40 & 0.27 \\
\hline 13 & 2.618 & 1.942 & 0.725 & 8.701 & 0 & 5.316 & 3.28 & 3.44 & 0.16 \\
\hline 14 & 2.618 & 1.942 & 0.725 & 0 & 0 & 10.790 & 3.14 & 3.12 & -0.02 \\
\hline 15 & 2.618 & 1.942 & 0.725 & 0 & 0 & 10.757 & 3.01 & 3.13 & 0.12 \\
\hline 16 & 2.954 & 2.364 & 1.291 & 0 & 0 & 5.756 & 4.25 & 4.26 & 0.01 \\
\hline 17 & 3.374 & 2.194 & 1.004 & 9.206 & 0 & 0 & 4.23 & 4.33 & 0.10 \\
\hline 18 & 2.618 & 1.942 & 0.725 & 17.306 & 0 & 0 & 3.71 & 3.75 & 0.04 \\
\hline 19 & 2.646 & 1.968 & 0.742 & 8.919 & 0 & 0 & 3.82 & 3.87 & 0.05 \\
\hline 20 & 2.853 & 2.159 & 0.869 & 8.787 & 0 & 0 & 4.03 & 3.94 & -0.09 \\
\hline 21 & 3.814 & 2.564 & 1.271 & 8.853 & 1.446 & 1.386 & 4.69 & 4.39 & -0.30 \\
\hline 22 & 4.745 & 3.558 & 2.232 & 25.824 & 0 & 0 & 5.17 & 5.27 & 0.10 \\
\hline 23 & 3.387 & 2.702 & 1.374 & 8.435 & 10.271 & 5.334 & 3.46 & 3.83 & 0.37 \\
\hline 24 & 3.415 & 2.729 & 1.394 & 0 & 10.716 & 5.416 & 3.34 & 3.94 & 0.60 \\
\hline 25 & 3.562 & 2.351 & 1.116 & 8.860 & 0 & 5.286 & 3.95 & 3.99 & 0.04 \\
\hline 26 & 4.274 & 3.061 & 1.749 & 17.333 & 10.272 & 0 & 4.41 & 4.70 & 0.29 \\
\hline 27 & 3.814 & 2.564 & 1.271 & 0 & 0 & 5.384 & 4.45 & 4.21 & -0.24 \\
\hline 28 & 2.954 & 2.364 & 1.291 & 0 & 0 & 5.624 & 4.36 & 4.27 & -0.09 \\
\hline 29 & 2.853 & 2.159 & 0.869 & 8.698 & 0 & 0 & 4.14 & 3.94 & -0.20 \\
\hline 30 & 3.387 & 2.702 & 1.374 & 8.454 & 10.298 & 5.333 & 3.67 & 3.83 & 0.16 \\
\hline 31 & 6.993 & 3.786 & 2.496 & 9.201 & 0 & 0 & 6.21 & 6.38 & 0.17 \\
\hline 32 & 3.338 & 2.656 & 1.339 & 17.047 & 10.147 & 0 & 3.97 & 4.12 & 0.15 \\
\hline 33 & 3.338 & 2.656 & 1.339 & 8.352 & 10.188 & 5.325 & 3.98 & 3.81 & -0.17 \\
\hline 34 & 1.968 & 1.756 & 0.478 & 8.632 & 0 & 0 & 3.62 & 3.40 & -0.22 \\
\hline 35 & 1.968 & 1.756 & 0.478 & 0 & 0 & 5.358 & 3.17 & 3.09 & -0.08 \\
\hline 36 & 2.618 & 1.942 & 0.725 & 0 & 0 & 10.742 & 3.06 & 3.13 & 0.07 \\
\hline
\end{tabular}




\section{Conclusion}

In this paper, molecular shape indices and topological state indices were analyzed. Six indices were selected to conduct the regression analysis with the toxicity of 36 anilines and phenolic compounds in leather material to daphnia magna. Then, a QSAR model was built which had good predictive ability of toxicity of aniline and phenolic compounds to daphnia magna. The coefficient of determination $\mathrm{R}_{\text {adj }}^{2}=0.885$ which was bigger than 0.8 , it showed good correlation in this model. The estimated values from this model were close to experimental values and their average relative error was $3.94 \%$ which totally met the requirement of experimental errors. From the estimated results, it could be seen that the biggest relative error, $18.08 \%$, came from the No.24 molecule para-aminoacetophenone. It might caused by the interaction of its azyl, carbonyl and middle ring which were on the intermediate positions and resulted in a relatively small value of toxicity. Moreover, it could be seen in the reference and predicted values of toxicity, when there were more than two groups on the benzene ring, the predicted error would increase. Especially when there were electron withdrawing groups, the negative charge of the benzene ring decreased and the toxicity of compounds increased. If there existed several groups and they interacted with each other, exceptional toxicity value would occur in this situation. Overall, the model in this paper has good predictive ability and good reliability. It is reliable to apply the method in prediction of toxicity of anilines and phenols.

\section{Acknowledgement}

This work was financially supported by National Natural Science Foundation of China (21472071) and Xuzhou City Green Key Laboratory Project (SYS2012009).

\section{References}

[1] W Dong, J F He, Y Q Wen, et al. West Leather, 35(2013)35-38

[2] B Shen. China Leather, 32(2003)29-31

[3] S C Yu, L M Xu, Y L Zhu, et al. Journal of Hydroecology,32(2011)131-134

[4] L Y Mo, H L Liu, S S Liu, et al. Asian Journal of Ecotoxicology,1(2006)259-264

[5] A Miyazaki , T Amano , H Saito , et al . Chemosphere,47(2002)65- 69

[6] X H Du. CIESC Journal, 61(2010)3059-3066

[7] X H Du. Food Science, 32(2011)218-221

[8] L Xu, Y P Wu, C Y Hu, et al. Science in China (Series B), 30(2000)1-7

[9] X L Zhang, Z X Zhou, X L Fang, et al. Chemical Research and Application, 25(2013)347-350

[10] S Y Shi, J F He, W Dong, et al. China Leather,42(2013)3-6

[11] Q N Hu, Y Z Liang, Y L Wang, et al. Computers and Applied Chemistry,20(2003) 386-390

[12] T Zhang, Y Z Liang, C X Zhao, et al. Chinese Journal of Analytical Chemistry, 34 (2006)1607-1610 